\title{
Building a Career as a Delivery Science Researcher in a Changing Health Care Landscape
}

\author{
Richard W. Grant, MD MPH and Julie A. Schmittdiel, PhD
}

Division of Research, Kaiser Permanente Northern California, Oakland, CA, USA.

J Gen Intern Med 30(7):880-2

DOI: $10.1007 / \mathrm{s} 11606-015-3178-9$

(c) Society of General Internal Medicine 2015

$\mathrm{H}$ ealth care in the U.S. is widely considered to be expensive and inefficient, and quality of care often falls short of evidence-based standards. These concerns have spurred concerted efforts to improve current models of health care delivery. Major examples of this effort to encourage higher quality care include implementation of the Affordable Care Act (ACA), which includes incentives to create accountable care organizations, and efforts by the Centers for Medicare and Medicaid Services (CMS) to increase "meaningful use" of electronic health records (EHRs).

Delivery science research seeks to overcome the barriers, understand the facilitators, and implement the innovations necessary to improve the process of health care. Delivery science has also been called "implementation science" or "translational research," because the overarching goal is to more effectively translate the evidence of clinical research into the practice of clinical care. Innovations examined or implemented by delivery science researchers include new health IT tools that leverage integrated electronic health records, new payment models to overcome some of the misaligned incentives of the traditional fee-for-service models, and patientcentered models of care that integrate individual patient preferences and values.

While the United States spends more than \$2 trillion on health care annually, less than $0.1 \%$ of this total (representing only $3.6 \%$ of the NIH budget) is currently devoted to research designed to improve how we deliver health care. ${ }^{1}$ This relative funding neglect may be changing, however, as exemplified by new funding programs within the National Institutes of Health (NIH) and Agency for Healthcare Research and Quality (AHRQ) focused on health care delivery, and the recently created Patient Centered Outcomes Research Institute (PCORI).

Conducting robust and rigorous research to improve health care delivery systems raises a number of novel research challenges. Addressing these challenges requires a well-trained

Published online January 16, 2015 research workforce with a new set of research skills. Training for delivery science shares some key dimensions with traditional health services research training, such as forming testable hypotheses, identifying and addressing sources of bias and confounding, and implementing rigorous biostatistical methods. In addition to this ability to access, manipulate, and understand large observational health care data sets, delivery scientists must also gain further skills in validating and inferring causality from these data in their efforts to design and evaluate effective interventions. This often requires applying novel statistical methods and using cutting-edge techniques such as natural language processing to glean useful information from EHR clinical notes; sophisticated modeling approaches such as marginal structural modeling or interrupted time-series; and innovative study designs including quasi-experimental methods, clustered randomization, and adaptive trial design. ${ }^{2}$

In addition, delivery science requires new skills that are not standard features of traditional research training programs. These skills can be summarized under three broad domains:

1) Understanding How Clinicians Create Health Care Data: Many health services training programs provide training in analytic methods involving very large data sets. With the widespread implementation of electronic health records (EHRs), there are now expanded opportunities to use clinical care data to improve health care. Beyond the expertise required to merge, clean, and validate these large quantities of clinical care data, delivery scientists must also be adept at understanding how and why different data elements are created in the flow of health care delivery. Understanding how these data are collected, and potentially playing a role in changing the data collection process when it may improve care, requires direct collaboration with the clinicians providing the patient care, the health IT programmers developing or modifying the EHR interfaces, and the quality improvement leaders establishing care goals. These important abilities to accurately analyze clinical data and influence clinical data collection require that delivery scientists develop skills in understanding the context in which clinical data are collected.

2) Developing Relationships with Stakeholders: Perhaps more so than any other field of clinical research, delivery science requires close working relationships with health care system stakeholders. ${ }^{3}$ Indeed, the success of any 
delivery science intervention will rely heavily on the ability to include and collaborate with system stakeholders. These stakeholders include practice leaders, managers, clinicians, and patients themselves. Health systems are enormously complex, and to successfully influence change in such a complex system, the delivery scientist must be closely embedded within the workings of the system itself. In addition, health systems have different goals from researchers. While scientists think in terms of grant funding cycles and significant $p$ values, our health care delivery stakeholders and collaborators necessarily focus on the day-to-day process of delivery care. Delivery science researchers should learn the importance of stakeholder engagement in all aspects of delivery science research, as well as the best methods for effectively aligning research, operational, and clinical agendas, as part of their training and development.

3) Resourcefulness in pursuing diverse and non-traditional funding sources: Sustaining a career as a delivery scientist often requires a funding portfolio that balances both "traditional" sources of research funding (e.g. NIH, AHRQ, PCORI) with non-traditional sources. These non-traditional sources can often come from the delivery system within which the scientist works. Being able to conduct both NIH-funded and operationally funded research requires that the investigator be able to respond nimbly to operational needs in a way that results in high quality and publishable science, while also providing useful results to the care delivery system on a much more rapid time line.

There are numerous challenges to this model of research career. Many systems use rapid "Plan-Do-StudyAct" (PDSA) cycles, for example, that are difficult to accommodate in more traditional research study designs. Questions of how to create a valid control group, how to encourage clinicians to adopt new tools, and how to generalize findings from a single system to other health care delivery settings must all be addressed for each project undertaken. Perhaps the greatest challenge in developing a delivery science research career is that clinical care is a moving target: interventions may need to be modified to align with changing delivery system goals and practices while the study is still ongoing. Given the often intensely "local" aspect of delivery science research, there may be challenges to publishing in top tier journals and obtaining funding from traditional NIH sources. The good news, however, is that there remains a tremendous need for skilled researchers to help in the sometimes painful but essential process of reconfiguring and modernizing our health care delivery systems. Primary care clinicians and other generalists in particular have a unique and important part to contribute to delivery science research, given their central role in overall patient care and in interfacing and coordinating with health care specialties. Demand for delivery scientists with cutting-edge research skills and expertise will continue to exist while there remains the strong need to control health spending, to more efficiently allocate health care resource, and to address the currently unmet potential for improved patient health and quality of life.

So how does one build a career as a delivery science researcher? Here's our advice: The key to a productive delivery science career is to find a problem or health care delivery issue for which you have a passion to solve. Beyond your own personal interest, this passion must also be linked to a larger problem (or set of problems) that is important to society as a whole. The need to balance your own research with the needs and concerns of health system stakeholders is fundamental to formulating good delivery science research questions. Listen to multiple voices and perspectives from different stakeholders. As one test, you should gauge others' enthusiasm when describing your research ideas. To be an effective delivery science researcher, your innovations need to be embraced by your stakeholders. Depending on the project, these stakeholders may include patients, clinicians, and/or system leaders. Without their enthusiasm, your chance of success is low. Also, obtain advanced training in the domains outlined above. New research fellowships (often embedded directly within health systems) are increasingly available to provide mentorship and training. ${ }^{4}$ As you proceed in your career from initial projects to next steps, begin to build a narrative arc for your work that connects your different projects to a unifying theme and links your findings to a future goal. Over the course of a successful career, you will likely have to flexibly be both an innovator and an evaluator. Have a vision for how your research can make the world (or at least health delivery systems) a better place.

This is an exciting time to be engaged in delivery science research, as pressures from both public and private payers are helping to transform our health system. There is a need for rigorous evaluation of ongoing changes and for creating new knowledge with innovative implementations. Given these changes, a core of well-trained researchers is needed to ask and answer the key questions facing the health care reform enterprise. Clinicians directly engaged in patient care now have the opportunity to obtain further training and to apply their own day-to-day clinical experiences to develop new avenues for innovation. We don't know what the U.S. health system will look like 5 or 10 years from now, but we do know it must change and that delivery scientists will be needed to help create this change.

Acknowledgements: R.W.G receives support from NIDDK (RO1 DK099108), NHLBI (R01 HL117939) and PCORI (SC14-140311992). J.A.S receives support from the Health Delivery Systems Center for Diabetes Translational Research [NIDDK P30-DK092924]. The authors wish to thank Dr. Tracy Lieu for comments on an earlier version of this manuscript. The authors report no conflicts of interest. 
Corresponding Author: Richard W. Grant, MD MPH; Division of ResearchKaiser Permanente Northern California, 2000 Broadway, Oakland, CA 94612, USA (e-mail: Richard.W.Grant@kp.org).

\section{REFERENCES}

1. https://www.academyhealth.org/files/publications/fundingreport.pdf. September 27, 2014
2. Gregg EW, Ali MK, Moore BA, Pavkov M, Devlin HM, Garfield S, Mangione CM. The importance of natural experiments in diabetes prevention and control and the need for better health policy research. Prev Chronic Dis. 2013;10:E14. doi:10.5888/pcd10.120145.

3. Schmittdiel J, Grumbach K, Selby Jv. Health Care System-Based Participatory Research: An approach for sustainable translational research and quality improvement. Ann Fam Med. 2010;8(3):256-259. PMCID: PMC2866724.

4. For example: http://www.dor.kaiser.org/external/fellowship/ and http:// www.academyhealth.org/Training/content.cfm?ItemNumber=13412. Accessed 14 January 2015. 